\title{
A Review of Image Compression Techniques
}

\author{
Rajandeep Kaur \\ Mtech Scholar (CSE) \\ CTIEMT, Shahpur Jalandhar
}

\author{
Pooja Choudhary \\ Assistant Professor (CSE) \\ CTIEMT, Shahpur Jalandhar
}

\begin{abstract}
An image compression method eradicates redundant and/or unrelated information, and resourcefully encodes leftovers. Practically, it is frequently essential to toss away both non redundant information and relevant information to attain the essential compression. In any case, the ploy is discovering methods that permit important information to be resourcefully extracted and represented. This paper copes with dissimilar compression methods for comprising the information in an image. The information can be compressed by means of Lossy techniques such as Quantization, Transform coding, Block Transform Coding or Lossless techniques such as Run Length Coding, Lossless Predictive Coding, Multi-resolution Coding. All these techniques have been discussed in this paper and the performance of any technique/method is analyzed on various parameters like MSE and PSNR.
\end{abstract}

\section{General Terms}

Lossless Compression, Lossy Compression, Image Formats, Quantization, Entropy coding.

\section{Keywords}

Discrete Cosine Transform (DCT), Discrete Wavelet Transform (DWT), Mean Square Error (MSE), Peak Signalto-Noise Ratio (PSNR)

\section{INTRODUCTION}

Image compression is reducing the size in bytes of a graphics file with no degradation in the quality of the image to an undesirable level. The decline in file size permits more images to be stored in a known amount of diskette or memory space. It also minimizes the time essential for images to be sent over the Internet or downloaded from network pages. There are more than a few different ways in which image files can be compressed. For Internet use, the two mainly common compressed graphic image formats are the JPEG format and the GIF format. The JPEG technique is more often used for photographs, while the GIF technique is commonly used for line art and other images in which geometric shapes are reasonably simple.

In general, three fundamental redundancies exist in digital images that follow.

\subsection{Psycho-visual Redundancy}

It is a redundancy equivalent to dissimilar sensitivities to all image signals by individual eyes. Therefore, eradicating some less relative significant information in our visual processing may be satisfactory.

\subsection{Inter-pixel Redundancy}

It is a redundancy equivalent to statistical dependencies amongst pixels, particularly between adjoining pixels.

\subsection{Coding Redundancy}

The uncompressed image is frequently coded with every pixel by a set length. For instance, an image with 256 gray scales is represented by an array of 8 -bit integers. By means of some variable length code schemes such as Huffman coding and arithmetic coding may create compression.

\section{IMAGE COMPRESSION STANDARD}

During the past two decades, a range of compression methods have been developed to address main challenges faced by digital imaging. These compression methods can be classified broadly as Lossy Compression Methods and Lossless Compression Methods.

\subsection{Lossy Compression methods}

Usually nearly all lossy compressors (Fig. 1) are three-step algorithms, each of which is in accordance with three types of redundancy mentioned above. The first phase is a transform to get rid of the inter-pixel redundancy to group information competently. Then a quantizer is applied to take out psychovisual redundancy to symbolize the packed information with as few bits as achievable. The quantized bits are then resourcefully encoded to get extra compression from the coding redundancy.

\section{Compressor}

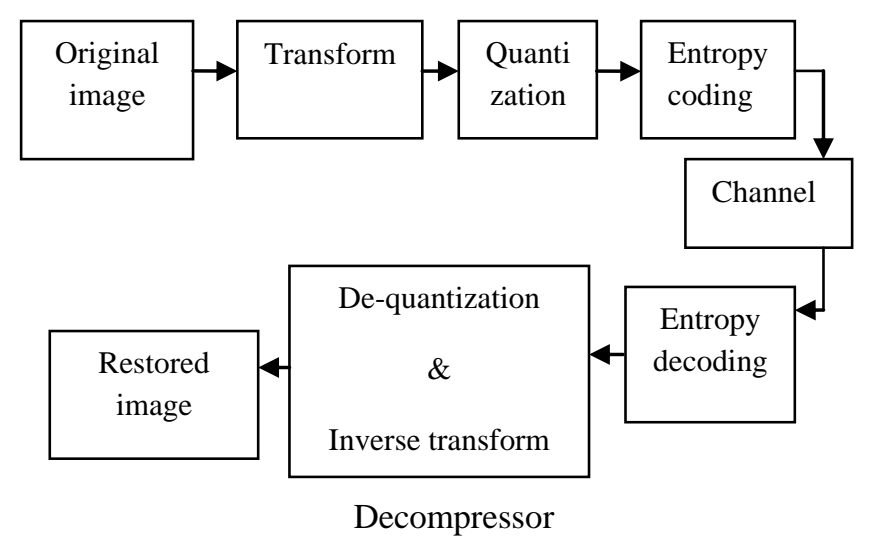

Fig 1: Lossy image compression

\subsubsection{Quantization}

Quantization is several to one mapping that replace a set of values with only one value. Scalar and vector quantization are two fundamental types of quantization. SQ (scalar quantization) performs various to one mapping on each value. VQ (vector quantization) replaces every block of input pixels with the index of a vector in the codebook, which is close to the input vector by using a few closeness dimensions. The decoder only receives each index and looks up the equivalent vector in the codebook.

Shannon first showed that VQ would outcome in a lower bit rate than SQ. But VQ suffers from a short of of generality, since the codebook ought to be taught on some set of initial images. As a result, the plan of the codebook will directly influence the bit rate and distortion of the compression [5]. 
Riskin et. al. offered variable-rate VQ design and applied it to MR images. Cosman et. al. used similar methods to compress $\mathrm{CT}$ and MR chest scans [1].

\subsubsection{Transform Coding}

Transform coding is a common method for lossy image compression. It employs a reversible and linear transform to decorrelate the actual image into a set of coefficients in transform domain. The coefficients are then quantized and coded successively in transform domain.

Several transforms are used in a range of applications. The discrete KLT (Karhunen-Loeve transform), which is based on the Hotelling transform, is most favorable with its information packing properties, but usually not practical since it is hard to work out. The DFT (discrete Fourier transform) and DCT (discrete cosine transform) fairly accurate the energy-packing efficiency of the KLT, and have further proficient implementation. In practice, DCT is used by the majority of practical transform systems since the DFT coefficients need two times the storage space of the DCT coefficients.

\subsubsection{Block Transform Coding}

In order to shorten the computations, block transform coding exploits correlation of the pixels inside a number of small blocks that divide the actual image. As a result, each block is altered, quantized and coded independently. This method, using square $8 \times 8$ pixel blocks and the DCT followed by Huffman or arithmetic coding, is utilized in the ISO JPEG (joint photographic expert group) draft international standard for image compression. The shortcoming of this method is the blocking (or tiling) artifacts become visible at soaring compression ratios.

As the acceptance of the JPEG standard, the algorithm has been the topic of great research. Collins studied the effects of a 10:1 lossy image compression method based on JPEG, with alterations to reduce the blocking artifacts. Baskurt used an algorithm analogous to JPEG to compress mammograms with a bit rate as low as 0.27 bpp (bits per pixel) while retaining recognition ability of pathologies by radiologists. Kostas used JPEG customized for use with 12-bit images and custom quantization tables to compress mammograms and chest radiographs.

Furthermore, the ISO JPEG commission is at present developing a new still-image compression standard called JPEG-2000 for deliverance to the marketplace by the end of the year 2000. The new JPEG-2000 standard is based upon wavelet decompositions collectively with a lot powerful quantization and encoding strategies such as embedded quantization and context-based arithmetic. It provides the potential for various advantages over the obtainable JPEG standard. Performance gains comprise improved compression efficiency at low bit rates for large images, while new functionalities consist of multi-resolution representation, scalability and embedded bit stream architecture, lossy to lossless progression, ROI (region of interest) coding, and a rich file format [3].

\subsection{Lossless Compression Methods}

Lossless compressors (Fig. 2) are generally two-step algorithms. The first step transforms the actual image to some other format in which the inter-pixel redundancy is minimized. The second step uses an entropy encoder to eliminate the coding redundancy. The lossless decompressor is an ideal inverse process of the lossless compressor [8].

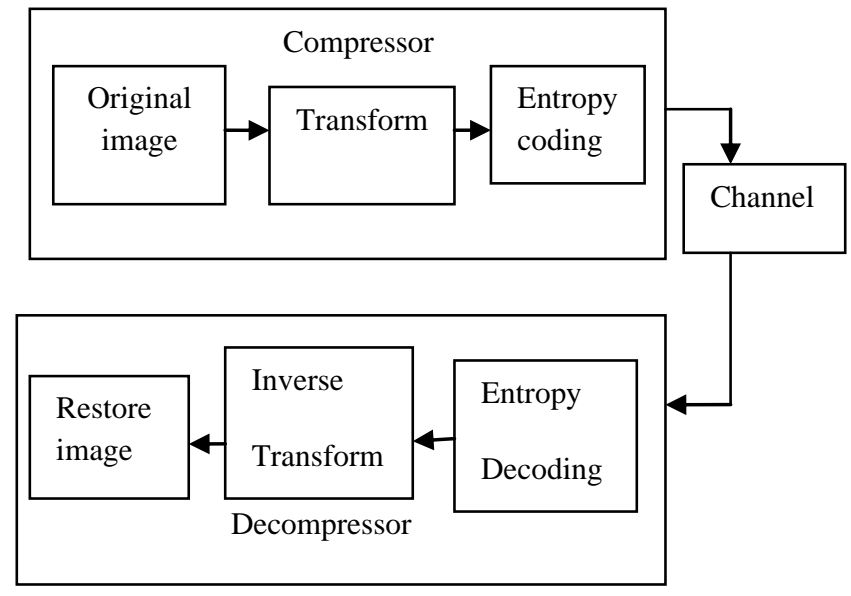

Fig 2: Lossless image compression

\subsubsection{Run length coding}

Run length coding replaces data by a (length, value) duo, where "value" is the recurring value and "length" is the quantity of repetitions. This method is especially winning in compressing bi-level images since the happening of a long run of a value is unusual in usual gray-scale images. A resolution to this is to decay the gray-scale image into bit planes and compress individual bit-plane separately. Efficient run-length coding technique is one of the variations of run length coding [7].

\subsubsection{Lossless Predictive Coding}

Lossless predictive coding predicts the value of every pixel by utilizing the values of its adjoining pixels. Consequently, each pixel is encoded with a prediction error somewhat than its actual value. Usually, the errors are a lot minor compared with the actual value so that less bits are obligatory to store them.

DPCM (differential pulse code modulation) is a predictive coding based lossless image compression scheme. It is too the base for lossless JPEG compression. A variation of the lossless predictive coding is the adaptive prediction that divides the image into blocks and calculates the prediction coefficients separately for every block to attain high prediction performance. It can too be combined with other scheme to get a hybrid coding algorithm with superior performance.

\subsubsection{Multi-resolution Coding}

HINT (hierarchical interpolation) is a multi-resolution coding method based on sub-samplings. It begins with a lowresolution edition of the actual image, and interpolates the pixel values to consecutively generate superior resolutions. The errors among the interpolation values and the real values are stored, along with the first low-resolution image. Compression is attained since both the low-resolution image and the error values can be stored with lesser bits than the actual image.

Laplacian Pyramid is an additional multiresolution image compression scheme developed by Burt and Adelson. It consecutively constructs inferior resolution editions of the actual image by down sampling so that the number of pixels reduces by a factor of two at every scale. The differences amongst consecutive resolution versions jointly with the lowest resolution image are stored and utilized to wholly reconstruct the actual image. But it cannot attain elevated 
compression ratio because the number of data values is improved by $4 / 3$ of the actual image size.

In general, the image is reversibly altered into a set of dissimilar resolution sub-images in multiresolution coding. Generally, it decreases the entropy of the image. Some kinds of tree representation could be worn to get more compression by exploiting the tree formation of the multiresolution methods.

\section{IMAGE REPRESENTATION FORMATS}

\subsection{Lossless image representation formats}

BMP (bitmap) is a bitmapped graphics design used within by the Microsoft Windows graphics subsystem (GDI), and used generally as a easy graphics file format on that stage. It is an uncompressed format [4].

PNG (Portable Network Graphics) (1996) is a bitmap image design that employs lossless data compression. PNG was formed to jointly improve upon and replace the GIF format with an image file format that does not need a patent license to use. It uses the DEFLATE compression algorithm, that uses a mixture of the LZ77 algorithm and Huffman coding. PNG chains palette based (with a palette defined in terms of the 24 bit RGB colors), grayscale and RGB images. PNG was planned for distribution of images on the internet not for qualified graphics and as such other color spaces [9].

TIFF (Tagged Image File Format) is a file format for primarily storing images, plus photographs and line art. It is one of the most admired and flexible of the current public domain raster file formats. Initially created by the company Aldus, together with Microsoft, for use with PostScript printing, TIFF is an admired format for high color depth images, alongside with JPEG and PNG. TIFF format is broadly supported by image-manipulation applications, and by scanning, faxing, word processing and other applications.

\subsection{Lossy image representation formats}

JPEG (Joint Photographic Experts Group) (1992) is an algorithm intended to compress images with 24 bits depth or grayscale images. It is a lossy compression algorithm. One of the distinctiveness that makes the algorithm extremely supple is that the compression rate can be attuned. If we compress a lot, more information will be lost, but the outcome image size will be lesser. With a lesser compression rate we obtain a better quality, but the size of the resulting image will be larger. This compression consists in making the coefficients in the quantization matrix bigger when we want added compression, and minor when we want less compression.

The algorithm is based in two visual effects of the human visual system. Firstly, humans are more responsive to the luminance than to the chrominance. Secondly, humans are added sensitive to changes in homogeneous areas, than in areas where there is more variation (higher frequencies). JPEG is the mainly used format for storing and transmitting images in Internet [10].

JPEG 2000 (Joint Photographic Experts Group 2000) is a wavelet-based image compression standard. It was formed by the Joint Photographic Experts Group committee with the intention of overriding their original discrete cosine transform based JPEG standard.

JPEG 2000 has superior compression ratios than JPEG. It does not undergo the uniform blocks, so distinctiveness of
JPEG images with very high compression rates. But it generally makes the image more blurred that JPEG.

\section{EVALUATION OF COMPRESSED IMAGE}

To calculate the quality of the image there are different parameters, normally used are Peak Signal to Noise Ratio and Mean Squared Error. In this section, different parameters are discussed.

The MSE is the cumulative squared error among the actual and the compressed image is calculated by [6]:

$$
\text { MSE }=\frac{1}{M N} \sum_{i=1}^{M} \sum_{j=1}^{N}(x(i, j)-y(i, j))^{2}
$$

The PSNR is a measure of the peak error between the actual and the compressed image is measured by:

$$
\operatorname{PSNR}(d B)=10 \log _{10}\left(\frac{255^{2}}{\mathrm{MSE}}\right)
$$

where $i(x, y)$ is the actual image, $z(x, y)$ is the approximated version (which is actually the decompressed image) and $M, N$ are the dimensions of the images. An inferior value for MSE means lesser error, and as seen from the inverse relation between the MSE and PSNR, this translates to a soaring value of PSNR.

\section{LOSSY vs LOSSLESS COMPRESSION}

The benefit of lossy methods over lossless methods is that in a few cases a lossy method can generate a much smaller compressed file than any recognized lossless method, while still meeting the necessities of the application.

Lossy methods are most frequently used for compressing sound, images or videos. The compression ratio (that is, the size of the compressed file compared to that of the uncompressed file) of lossy video codes are almost always far better to those of the audio and still-image equivalents. Audio can be compressed at 10:1 with no perceptible loss of quality; video can be compressed vastly with small visible quality loss, eg 300:1. Lossily compressed still images are often compressed to $1 / 10$ th their actual size, as with audio, but the quality loss is added noticeable, especially on closer examination.

When a user acquires a lossily-compressed file, (for example, to decrease download-time) the retrieved file can be quite unusual from the actual at the bit level while being indistinguishable to a person ear or eye for most practical purposes. Many techniques focus on the idiosyncrasies of the human anatomy, taking into consideration, for example, that the human eye can see only convinced frequencies of light. The psycho-acoustic model describes how sound can be greatly compressed without degrading the apparent quality of the sound. Flaws caused by lossy compression that are noticeable to the human eye or ear are known as compression artifacts.

Lossless compression algorithms usually make use of statistical redundancy in such a way as to represent the sender's data more concisely, but nevertheless perfectly. Lossless compression is likely because nearly all real-world data has statistical redundancy. For example, in English text, the letter ' $\mathrm{e}$ ' is a lot more common than the letter ' $\mathrm{z}$ ', and the 
probability that the letter ' $q$ ' will be followed by the letter ' $z$ ' is very less.

Another kind of compression, called lossy data compression, is feasible if some loss of fidelity is tolerable. For example, a person viewing a picture or television video scene might not notice if some of its premium details are detached or not represented entirely. Similarly, two clips of audio may be perceived as the same to a listener even though one is missing details found in the other. Lossy data compression algorithms bring in relatively small differences and represent the picture, video, or audio using lesser bits.

Lossless compression methods are reversible so that the actual data can be reconstructed, while lossy methods accept some loss of data in order to attain higher compression. However, lossless data compression algorithms will constantly fail to compress a few files; indeed, any compression algorithm will necessarily fail to compress any data containing no discernible patterns. Attempts to compress data that has been compressed already will therefore generally result in an expansion, as will attempts to compress encrypted data.

In practice, lossy data compression will also come to a point where compressing again does not work, although an extremely lossy algorithm, which for example always removes the last byte of a file, will always compress a file up to the point where it is empty.

\section{CONCLUSION}

In foregoing years, image compression has become fascinated and vital field. In these past years, many researchers gave many techniques of image compression. In this paper, foremost compression techniques and the quality estimation parameters are reviewed. And is conclude that two types of techniques can be used for compression. In lossless techniques image quality is maintained as original after decompression but not too much compression ratio can be achieved. But in case of lossy techniques compression ratio can be achieved more than lossless but at the cost of image quality.

\section{REFERENCES}

[1] P. C. Cosman, K. L. Oehler, E. A. Riskin and R. M. Gray, "Using vector quantization for Image Processing", IEEE, Volume 81, Issue 9, pp. 1326-1341, 1993.
[2] N. Memon, X. Wu, V. Sippy, and G. Miller. An interband coding extension of the new lossless jpeg standard. Proc. SPIE Visual Communications and Image Processing, 3024, pp. 47-58, January 1997.

[3] D. S. Cruz, R. Grosbois and T. Ebrahimi, "JPEG 2000 performance evaluation and assessment", Signal SProcessing: Image Communication, Volume 17, Issue 1, pp. 113-130, 2002.

[4] Dr T. Meyyappan, SM.Thamarai and N. M. Nachiaban, "Lossless Digital Image Compression Method For Bitmap Images", The International Journal of Multimedia \& Its Applications (IJMA) Vol.3, Issue .4, November 2011

[5] D. Singh, S. K. Ranade, "Comparative Analysis of Transform based Lossy Image Compression Techniques" International Journal of Engineering Research and Applications (IJERA), Vol. 2, Issue 5, pp.1736-1741, October 2012.

[6] Dr. B E. Reddy and K. V. Narayana, "A Lossless Image Compression Using Traditional And Lifting Based Wavelets", Signal \& Image Processing : An International Journal (SIPIJ) Vol.3, No.2, April 2012.

[7] G. Vijayvargiya, Dr. S. Silakari and Dr. R. Pandey, "A Survey: Various Techniques of Image Compression, International Journal of Computer Science and Information Security, Vol. 11, No. 10, October2013.

[8] M. Kaur and G. Kaur, "A Survey of Lossless and Lossy Image Compression Techniques", International Journal of Advanced Research in Computer Science and Software Engineering, Volume 3, Issue 2, February 2013.

[9] Firas A. Jassim, "Increasing Compression Ratio in PNG Images by k-Modulus Method for Image Transformation", International Journal of Advanced Research in Computer Science and Software Engineering, Volume 3, Issue 6, June 2013.

[10] B. Gupta, M. Gupta and B. Chadha, "Image Compression Technique under JPEG by Wavelets Transformation", International Journal of Advanced Research in Computer Science and Software Engineering, Volume 4, Issue 6, June 2014. 